The AstrophysicAl JouRnAL, 559:452-462, 2001 September 20

(C) 2001. The American Astronomical Society. All rights reserved. Printed in U.S.A.

\title{
ON THE TEMPORAL RELATIONSHIP BETWEEN CORONAL MASS EJECTIONS AND FLARES
}

\author{
J. Zhang, ${ }^{1}$ K. P. Dere, ${ }^{2}$ R. A. Howard ${ }^{2}$ M. R. Kundu, ${ }^{3}$ AND S. M. White ${ }^{3}$ \\ Received 2000 November 27; accepted 2001 May 29
}

\begin{abstract}
The temporal relationship between coronal mass ejections (CMEs) and associated solar flares is of great importance to understanding the origin of CMEs, but it has been difficult to study owing to the nature of CME detection. In this paper, we investigate this issue using the Large Angle and Spectrometric Coronagraph and the EUV Imaging Telescope observations combined with GOES soft X-ray observations. We present four well-observed events whose source regions are close to the limb such that we are able to directly measure the CMEs' initial evolution in the low corona $\left(\sim 1-3 R_{\odot}\right)$ without any extrapolation; this height range was not available in previous space-based coronagraph observations. The velocity-time profiles show that kinematic evolution of three of the four CMEs can be described in a three-phase scenario: the initiation phase, impulsive acceleration phase, and propagation phase. The initiation phase is characterized by a slow ascension with a speed less than $80 \mathrm{~km} \mathrm{~s}^{-1}$ for a period of tens of minutes. The initiation phase always occurs before the onset of the associated flare. Following the initiation phase, the CMEs display an impulsive acceleration phase that coincides very well with the flares' rise phase lasting for a few to tens of minutes. The acceleration of CMEs ceases near the peak time of the soft X-ray flares. The CMEs then undergo a propagation phase, which is characterized by a constant speed or slowly decreasing in speed. The acceleration rates in the impulsive acceleration phase are in the range of 100-500 $\mathrm{m} \mathrm{s}^{-2}$. One CME (on 1997 November 6, associated with an X9.4 flare) does not show an initiation phase. It has an extremely large acceleration rate of $7300 \mathrm{~m} \mathrm{~s}^{-2}$. The possible causes of CME initiation and acceleration in connection with flares are explored.
\end{abstract}

Subject headings: Sun: corona - Sun: coronal mass ejections (CMEs) - Sun: flares

\section{INTRODUCTION}

Coronal mass ejections (CMEs) and flares have been major research subjects in solar physics and space science for several decades. These two energetic solar phenomena are believed to be the causes of many geoeffective activities such as geomagnetic storms. Since they have often been found to be associated with each other, the cause-effect relationship between them has long been pursued in order to understand the physical mechanisms involved (Kahler 1992; Gosling 1993; Dryer 1996; Hundhausen 1999). However, the controversy continues to exist. It seems that the view of the relationship has been shifting from a traditional flare-cause scenario to a CME-cause scenario. The difficulty in solving the controversy observationally is largely due to the lack of high-quality CME observations in the low corona, where CMEs are initiated and accelerated. Using advanced Solar and Heliospheric Observatory (SOHO) observations, in this paper we intend to look into this issue by investigating detailed temporal relationship between CMEs and flares.

Statistical studies, which have been performed for almost every major coronagraph, have revealed that CMEs are intimately associated with flares, as well as other surface phenomena such as eruption filaments. The association is mainly based on a time-window criterion: the extrapolated departure time of a CME from the solar surface falls into a time window with respect to the onset time of the corresponding flare, usually referring to soft X-ray flares

\footnotetext{
${ }^{1}$ Center for Earth Observing and Space Research, School of Computational Science, George Mason University, Fairfax, VA 22030.

${ }^{2}$ E.O. Hulburt Center for Space Research, Naval Research Laboratory, Washington, DC 20375.

${ }^{3}$ Astronomy Department, University of Maryland, College Park, MD 20742.
}

observed by GOES. Munro et al. (1979) found an association rate of $16 \%$ : of 77 CMEs observed by the High Altitude Observatory's white-light coronagraph (inner occulter $>2 R_{\odot}$ ) on board Skylab, there were 12 associated GOES $\mathrm{X}$-ray flares (stronger than $\mathrm{C} 1$, time window \pm 30 minutes). Webb \& Hundhausen (1987) found an association rate of $33 \%$ : 19 GOES X-ray flares (stronger than C1, time window \pm 90 minutes) were associated with 58 CMEs observed by the High Altitude Observatory's Coronagraph/Polarimeter (inner occulter $>1.6 R_{\odot}$ ) on the Solar Maximum Mission $(S M M)$ satellite. Sheeley et al. (1983) presented an association rate of 50\% among 125 major CMEs observed by the Naval Research Laboratory Solwind White-Light Coronagraph on board the P78-1 satellite (inner occulter $>2.5 R_{\odot}$, GOES X-ray flares greater than $\mathrm{C} 1$, time window \pm 30 minutes). For $S M M$ coronagraph observations from 1984 to 1986, St. Cyr \& Webb (1991) found an association rate of 48\% (35 GOES X-ray events with 73 CMEs, flares greater than $\mathrm{C} 1$, time window \pm 90 minutes). For a more comprehensive study of $S M M$ observations, Harrison (1995) also found an association rate of $48 \%$ (151 CMEs, flares greater than $\mathrm{B} 1$, time window \pm 120 minutes). Considering that roughly half the number of CMEs are initiated from the backside of the solar disk so that accompanying X-ray flares may not be detected by GOES observations (except those events so close to the limb that post-flare X-ray loops rise above the limb), the actual association rate should be determined by multiplying the above numbers by a factor of nearly 2 . Therefore, it is justified to conclude that a significant percentage of CMEs are accompanied by X-ray flares, although the real association rate varies with selection criteria.

There are several additional observational facts regarding the relationship between CMEs and flares based on statistical studies. The probability of association seems to 
increase monotonically with the duration of the flare (Sheeley et al. 1983; Harrison 1995). Kahler, Sheeley, \& Liggett (1989) revealed a good relationship between flare duration and CME angular width. Regarding the spatial relationship, the locations of flares on the solar disk could be anywhere within the CME angular span; flares are neither centered below the CMEs, nor do they lie preferentially at CME legs (Harrison 1986; Kahler et al. 1989).

The apparent association between CMEs and flares suggests that there is a physical link between them. However, that physics is poorly known. Coronagraphs, the primary tool to observe CMEs, are mostly externally occulted white-light coronagraphs. They are usually not able to observe the solar disk and low corona (e.g. $<2 R_{\odot}$ ), because they have to use an occulter to block the otherwise dominant light from solar disk. As a consequence, the observations are not able to provide views of early evolution of CMEs in the low corona but provide morphology in the middle and high coronae. A CME appearing in a coronagraph is thus spatially displaced from the position where the flare can be observed. The time at which a coronagraph sees the CME is also displaced from the flare onset time. The CME onset time, which has to be extrapolated using the observations above the occulting disk, is found to be randomly located within windows tens of minutes wide around the flare onset time (Harrison 1995). This scattering distribution is very likely due to the uncertainty in the extrapolation method, which assumes a constant CME velocity behind the occulter and zero altitude of CME onset position; both assumptions are not appropriate and lead to the controversial result. Prior to the $\mathrm{SOHO}$ observations, the ground-based MK3 K-coronameter of the High Altitude Observatory (occulter from 1.3 to $2.2 R_{\odot}$; Fisher \& Poland 1981) provides a viable tool to observe CMEs at low coronal heights. By examining individual events based on combined MK3 and SMM observations, it has been demonstrated that CMEs start almost simultaneously with the accompanying flares (Maxwell, Dryer, \& McIntosh 1985; Dryer 1994), or CMEs onset a few minutes earlier than the flare onset time (Hundhausen 1999).

In this paper, we investigate the temporal relationship between CMEs and flares in a more comprehensive way by making use of observations with the Large Angle and Spectrometric Coronagraph (LASCO; Brueckner et al. 1995), which covers the corona from 1.1 to $30 R_{\odot}$, and the EUV Imaging Telescope (EIT; Delaboudinière et al. 1995), which observes the Sun's disk and corona up to $1.5 R_{\odot}$; both instruments are on board the $\mathrm{SOHO}$ spacecraft. The combined EIT and LASCO observations provide an unprecedentedly complete view of the initiation, acceleration, and subsequent propagation of CMEs. Using these observations, we are not only able to determine the longargued relative onset time between CMEs and flares without resorting to any extrapolation, but we are also able to find out how a CME changes speed with respect to different phases of the flare, which provides an additional and essential insight into the relationship. We have identified four well-observed events suitable for this study after searching through a large portion of the database. In $\S 2$, we present the observations. In $\S 3$, we present our analysis of the selected events. In $\S 4$, we discuss the implications of our results. Conclusions are given in $\S 5$.

\section{OBSERVATIONS}

The LASCO instrument consists of a set of three nested coronagraphs with overlapping and concentric fields of view: C1 (1.1-3 $\left.R_{\odot}\right), \mathrm{C} 2\left(2-6 R_{\odot}\right)$, and C3 $\left(4-30 R_{\odot}\right)$. C1, an internally occulted coronagraph, performs high-resolution

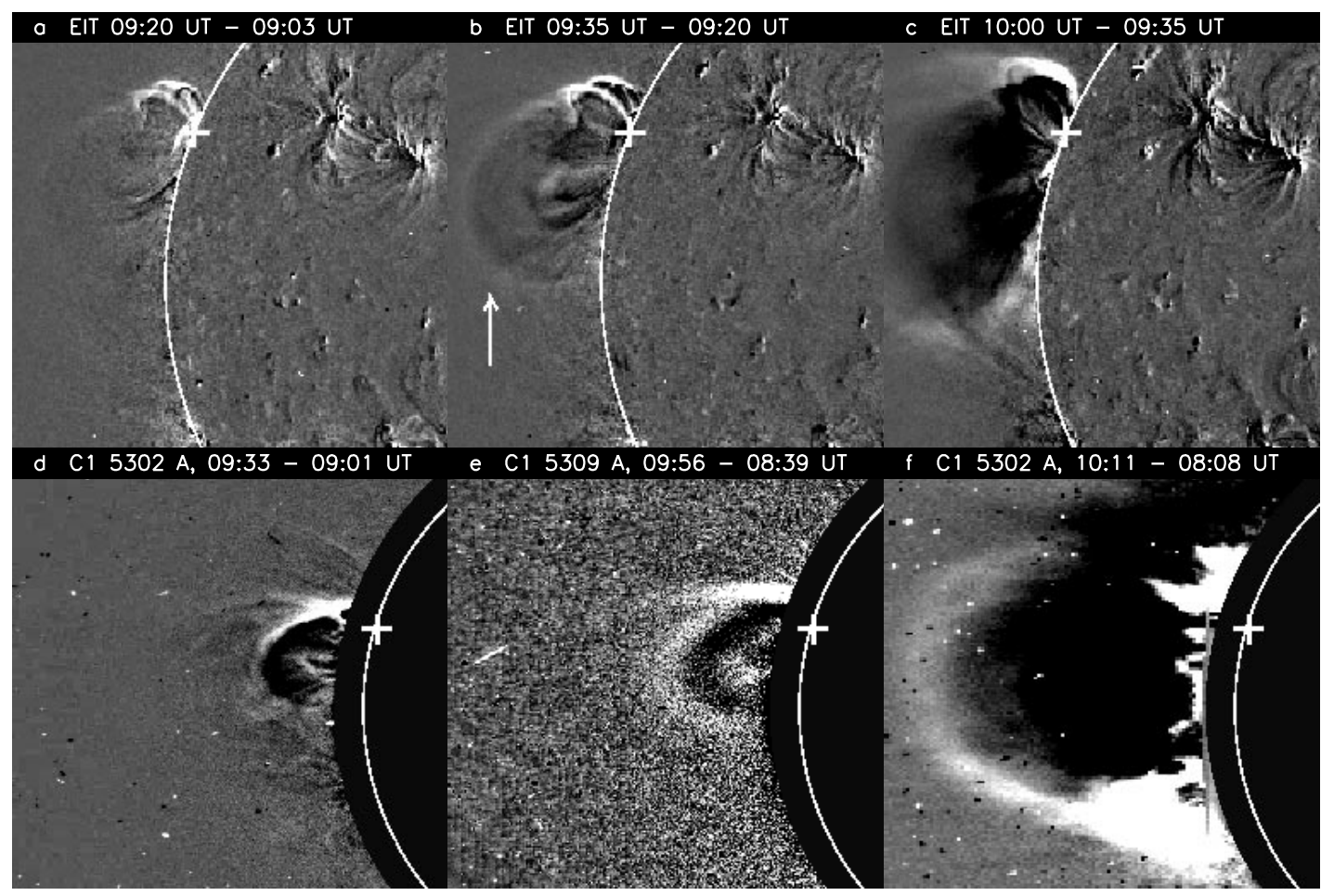

FIG. 1.- Running difference images of EIT $\lambda 195$ (upper three panels) and LASCO/C1 (lower three panels), which show early evolution of the CME on 1998 June 11. Their timings are indicated at the top of each panel. The white circle in each panel indicates $1.0 R_{\odot}$ solar limb. The plus sign in each panel indicates the position of the corresponding flare. The arrow in $(b)$ points at the CME front. 
imaging spectroscopy of the corona. A Fabry-Pérot interferometer and a set of blocking filters are used to isolate selected coronal emission lines. The images are mostly taken in the few strongest optical spectral forbidden lines in the corona, e.g., the green line of Fe XIV at $5303 \AA$ and the red line of $\mathrm{Fe} x$ at $6374 \AA$. The $\mathrm{C} 1$ images, which have an unprecedented view of the low corona (Plunkett et al. 1997; Schwenn et al. 1997), provide key information on the early evolution of CMEs. C2 and C3 are traditional externally occulted white-light coronagraphs that observe Thomsonscattered visible light through a broadband filter. They provide an extensive view of CME evolution in the middle and high coronae. The EIT instrument, a normal-incidence telescope with multilayer-coated mirrors, makes images of the Sun in four narrow EUV channels centered at 171, 195, 284 , and $304 \AA$, which selectively observe strong spectral lines formed by $\mathrm{Fe} \mathrm{IX} / \mathrm{x}, \mathrm{Fe} \mathrm{XII}, \mathrm{Fe} \mathrm{XV}$, and $\mathrm{He}$ II, respectively. EIT images have full view of the solar disk and the corona extending up to $1.5 R_{\odot}$. Therefore, EIT images provide us a view of the coronal environment of CMEs and their early evolution as well. C1 and EIT, which are sensitive to emission lines of highly ionized ions, observe the coronal structure composed of plasma in millions of degrees. On the other hand, $\mathrm{C} 2$ and $\mathrm{C} 3$, sensitive to the white light scattered by free electrons, observe electron density structure in the corona. The three LASCO coronagraphs and EIT started observations shortly after the launch of $\mathrm{SOHO}$ on 1995 December 2. They continue to operate up to the present date except for the $\mathrm{C} 1$ instrument, which has been out of function since the $\mathrm{SOHO}$ interruption in 1998 June. Thus, studies exploiting C1 observations are limited to the period from 1996 January through 1998 June.

The temporal profiles of flares are from 1 to $8 \AA$ soft X-ray flux provided by detectors on the GOES satellites, which continuously record the full-disk-integrated X-ray flux with a temporal resolution of $3 \mathrm{~s}$. The locations of flares can be determined from EIT images. Flare locations can also be found in the Solar Geophysics Data Reports based on ground-based optical $\mathrm{H} \alpha$ imaging observations. Note that the magnitude of the GOES X-ray flare is expressed in units of watts per meter ${ }^{2}$ and classified into classes designated by letters $\mathrm{A}, \mathrm{B}, \mathrm{C}, \mathrm{M}$, and $\mathrm{X}$, with flux starting at $10^{-8}, 10^{-7}, 10^{-6}, 10^{-5}$, and $10^{-4} \mathrm{~W} \mathrm{~m}^{-2}$, respectively. The $\mathrm{X}$-ray flux is very sensitive to enhanced thermal emission in the corona. As a matter of fact, the number of X-ray flares (>B1 level) is several times as large as the number of CMEs observed during the same period (Harrison 1995). While GOES soft X-ray observations are readily available without interruption, it is also desirable to include hard X-ray observations in the study, since a hard X-ray is a better indicator of impulsive energy release in the corona. For one of the events presented in this paper, a hard X-ray counterpart was observed by the Hard X-Ray Telescope (HXT) on board the $Y O H K O H$ spacecraft, and we include it in the presentation.

When a sufficient number of coronagraph images are made during the CME/flare time, we can straightforwardly determine the onset time and acceleration of a CME with respect to the accompanying flare. The cadence of LASCO and EIT observations limits this ability to some extent. The typical rise phase of a flare lasts a few minutes to tens of minutes. On the other hand, the EIT $\lambda 195$ channel and C2 has a rather regular cadence of $\sim 20$ and $\sim 30$ minutes, respectively. $\mathrm{C} 1$ has a variable cadence from a few minutes to 30 minutes depending on the observing sequence in use. The cadence of the regular green-line observations of $\mathrm{C} 1$ is about 30 minutes. But in fact, $\mathrm{C} 1$ takes about 150 images daily on many days during its period of operation, which results in an average cadence of 10 minutes; these images include observations of spectral scans of the same line and other strong lines. In addition to the concern of the cadence of CME observations, we are limited to CME events whose source regions are close to the limb, since only these events can be well observed by the $\mathrm{C} 1$ coronagraph. CME features are rarely detected by $\mathrm{C} 1$ if the source region is close to the disk center. Furthermore, we use only those events whose source region is on the front side of the disk or above the limb, in order to know the pre-CME coronal environment and to make unambiguous association with flares. The source regions of CMEs are determined by EIT observations, using signatures such as dimmings and post-flare loops. Searching through the $\mathrm{C} 1$ database, we have found several dozen C1 CMEs out of about 800 CMEs observed by $C 2$ and $C 3$ during the period of 1996 January-1998 June. Most of the C1 CMEs have only one or two frames. But we have fortunately found four events with sufficient cadence to carry out a study on their detailed temporal relationship with flares. They are observed on 1997 February 23, 1997 May 16, 1997 November 6, and 1998 June 11, respectively.

\section{ANALYSIS OF CME/FLARE EVENTS}

\subsection{June 11 Event}

The CME of 1998 June 11 is associated with a GOES M1.4 class flare. Its source region is a complex system of coronal loops above the northeastern limb that are rooted in NOAA Active Region 8243. The position of this active region is roughly $\mathrm{N} 18^{\circ}, \mathrm{E} 92^{\circ}$ (or latitude north $18^{\circ}$, longitude $2^{\circ}$ behind the east limb) as extrapolated from its positions in magnetogram images obtained a few days later when this active region had rotated onto the visible disk. To illustrate the CME evolution, we display the EIT (upper three panels) and $\mathrm{C} 1$ (lower three panels) running difference images of the region of interest during the eruption in Figure 1; the white circle indicates the $1.0 R_{\odot}$ solar limb, and the plus sign indicates the flare position as determined from the post-flare loop system seen above the limb later on. Note that we have not displayed the $\mathrm{C} 2$ and $\mathrm{C} 3$ images since we are mainly interested in the early evolution of CMEs, not the later propagation.

In EIT, the CME is first apparent at 09:35 UT with its front seen as a semicircular bright rim in the image (Fig. $1 b$, arrow); in an earlier EIT image at 09:20 UT (Fig. 1a), the CME front has not been fully formed, at least to the sensitivity of EIT observations. It seems that the CME front is created by expansion of the envelope of the loop arcade in the active region. The CME front has an initial height of $0.34 R_{\odot}$, or $240,000 \mathrm{~km}$ above the limb. It has a dimension of $300,000 \mathrm{~km}$ between its northern and southern footpoints. At 10:00 UT when the next EIT frame was taken, the CME front is already out of the field of view of EIT (Fig. $1 c$ ) but is in the field of view of $\mathrm{C} 1$ at that time.

The three $\mathrm{C} 1$ images in Figure 1 illustrate how the CME evolved in the low corona. The CME ascended while expanding laterally as well. At 09:56 (Fig. 1e) and 10:11 UT (Fig. $1 f$ ), the CME front has achieved heights of 1.53 and $2.15 R_{\odot}$ (heliocentric distance), respectively. The images in Figures $1 d$ and $1 f$ are from observations of green-line 
coronae at $5302 \AA$, while the image in Figure $1 e$ is from an off-green-line observation at $5309 \AA$. We have analyzed all available $\mathrm{C} 1$ images of different spectral lines in order to achieve a better cadence. During the eruption period, there are eight images in total taken by $\mathrm{C} 1$ from $09: 20$ to $10: 14$ UT. The overall cadence of $\mathrm{C} 1$ is usually better than EIT's, although the cadence for a fixed wavelength is longer and varies significantly with wavelength. In order to enhance weak changes in coronal features, we use difference images obtained by subtracting sequential images taken at the same wavelength with the same frame size.

We now investigate the temporal relationship between the CME and the flare. In the upper panel of Figure 2, the height-time plot of the CME (dotted line) observed with three LASCO coronagraphs (asterisks, triangles, and squares) is shown along with the plot of the GOES soft X-ray flux (solid line). The signs are simply linked by (but not fitted to) the dotted line. The numbers on the $Y$-axis represent the apparent value of the CME height, while the $\mathrm{X}$-ray flux is displayed in an arbitrary unit to fit in the
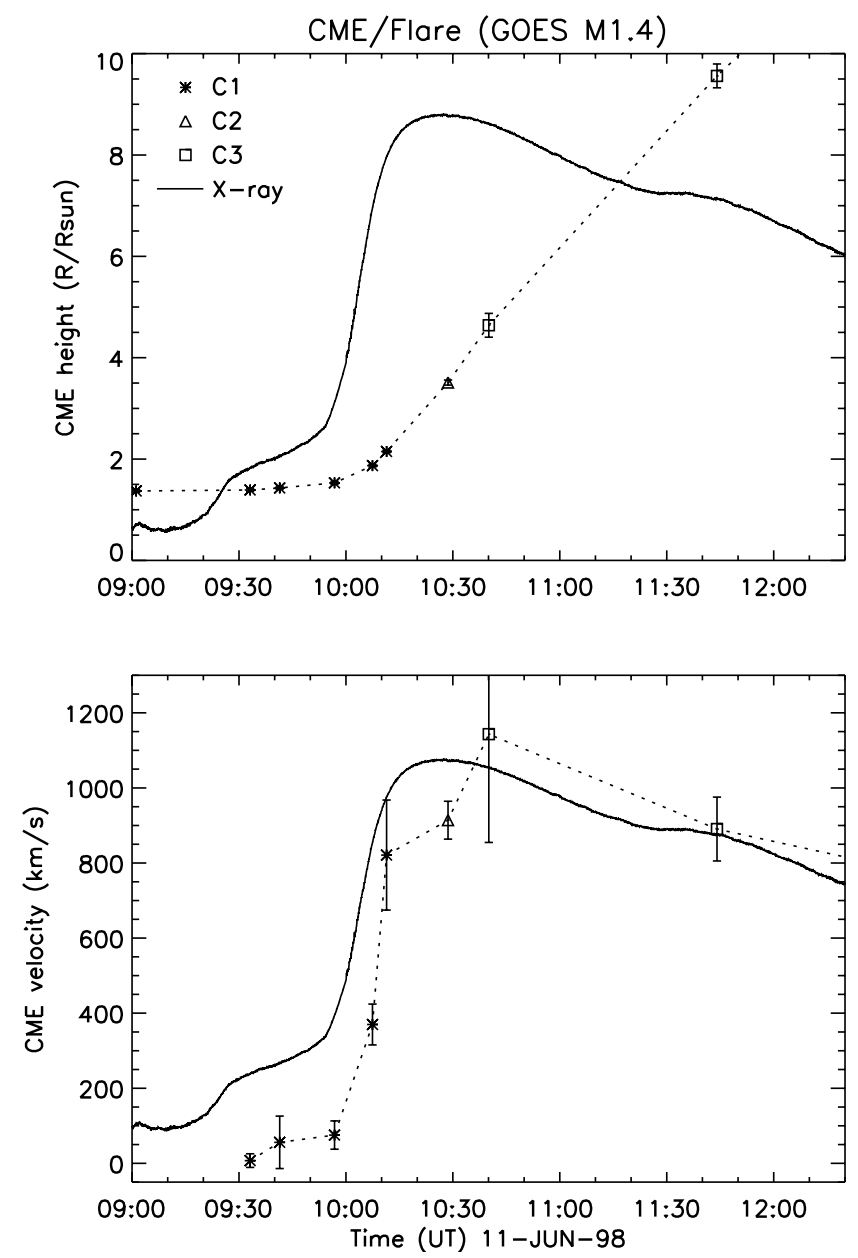

FIG. 2.-Upper panel: height-time profile (dotted line) of the CME on 1998 June 11 vs. the temporal profile of GOES X-ray flux of corresponding M1.4 flare (solid line). The $Y$-axis denotes the CME height (heliocentric distance of CME front in units of $R_{\odot}$ ), and the X-ray flux is displayed as an arbitrary unit. The CME heights, which are measured in a set of LASCO $\mathrm{C} 1, \mathrm{C} 2$, and $\mathrm{C} 3$ images are designated by asterisks, triangles, and squares, respectively; the dotted line connects these symbols. Lower panel: CME velocity-time profile (dotted line) vs. the flare temporal profile (solid line). Velocities are derived using the height difference between the images at the given time and the previous image. panel. The peak of the X-ray flare is M1.4, and the background is at B4 level. The height of the CME at a given snapshot image is defined by the highest point of the leading edge. The leading edge of the CME, as seen as a semicircular loop shape in both the emission-line images of EIT and $\mathrm{C} 1$ and white-light images of $\mathrm{C} 2$ and $\mathrm{C} 3$, is believed to be a density feature of the CME. Since the CME front is unique for a particular CME, we believe that we are tracking the same feature of the CME in different coronal images. We have also cross-checked some near-simultaneous EIT-C1 image pairs as well as C1-C2 image pairs and found that the CME front is identical although the internal structure may appear different. The same tracking rule has been applied to other events presented in this paper. The uncertainty of measuring CME height is estimated to be less than 8 pixels in each image, depending on the sharpness of the leading edge. In terms of solar radius, the uncertainty is $0.02,0.05,0.10$, and $0.47 R_{\odot}$ for EIT, C1, C2, and C3, respectively; the error bars attached to each sign indicate the uncertainty of measured height.

In order to illustrate how the CME speed changes with respect to the flare, we make a velocity-time plot of the CME (dotted line) along with the temporal profile of the flare (solid line) in the lower panel of Figure 2. The velocity is derived by using heights measured in two adjacent observations instead of a polynomial fitting to the height-time curve. Note that the widely used polynomial fitting method is not applicable here since the CME undergoes fast velocity changes, and there is not a sufficient number of measurements during the phase of fast change. The velocity thus calculated is an average velocity during the period of two adjacent observations. The uncertainty in calculating CME velocity (denoted by error bars on the signs) is caused by the uncertainty in measuring CME height; the shorter the cadence, the larger the uncertainty of such calculated velocity. The numbers on the $Y$-axis represent the value of CME velocity, while the X-ray flux is displayed in arbitrary units.

As seen in the lower panel of Figure 2, it is quite apparent that the CME velocity profile is similar to the flare X-ray profile. For the sake of clarity, we replot the profiles in Figure 3 and divide and label them in different phases. The soft X-ray profile of the flare can be roughly divided into three phases: a preflare phase of 39 minutes from 09:18 to 09:57 UT, a rise phase of 30 minutes from 09:57 to 10:27 UT, and a decay phase of more than $10 \mathrm{hr}$ after 10:27 UT. The CME velocity profile can also be divided into three phases, which for convenience we call the initiation phase, the impulsive acceleration phase, and the propagation phase that correspond to the three phases of the X-ray time profile, respectively. In the initiation phase (from 09:33 to 09:56 UT), the newly formed CME front underwent a slow ascension with velocity less than $75 \mathrm{~km} \mathrm{~s}^{-1}$. At the end of this phase, the CME front had only reached a height of 1.53 $R_{\odot}$ from its original height of $1.34 R_{\odot}$. Following the initiation phase, the CME underwent a rapid acceleration. From 09:56 to 10:40 UT, the CME speed jumped from 75 to $1150 \mathrm{~km} \mathrm{~s}^{-1}$; in the meantime, the CME height increased from 1.53 to $4.64 R_{\odot}$. This impulsive acceleration phase of the CME coincides very well with the rise phase of the flare. Later, the CME maintained a relatively constant speed of about $800 \mathrm{~km} \mathrm{~s}^{-1}$ until it went out of the LASCO field of view.

It is useful to quantify the impulsive acceleration of CMEs in order to make comparative studies among various 


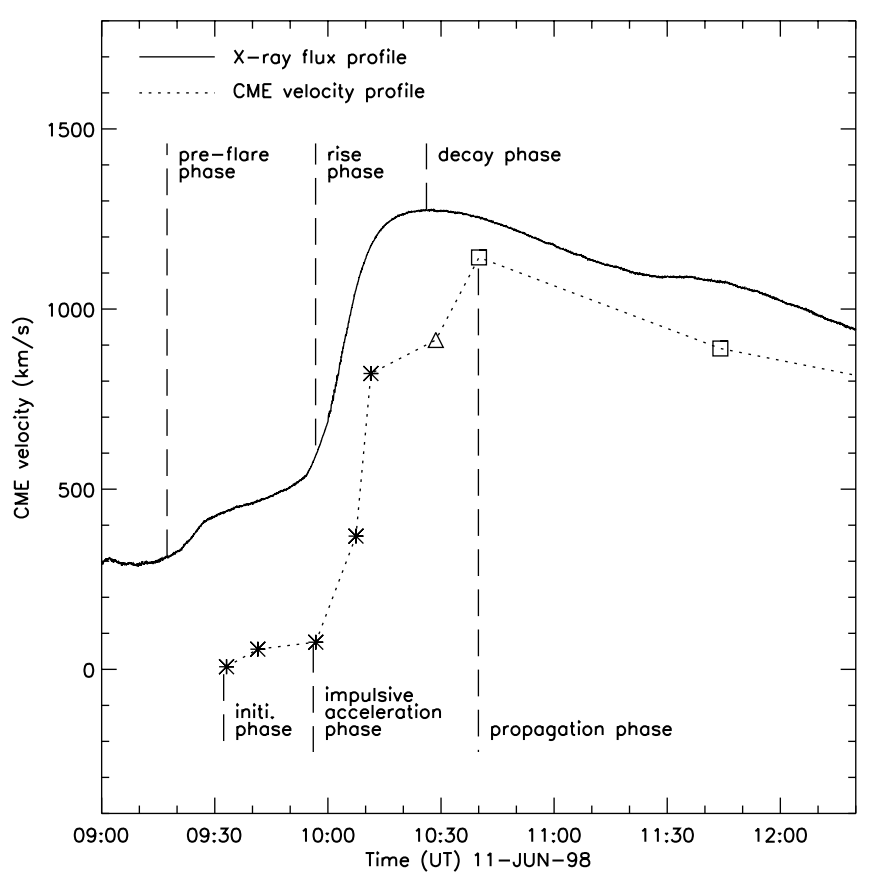

FIG. 3.-Illustration of three phases of the CME velocity-time profile (dotted line) and the flare soft X-ray flux temporal profile (solid line) for the event on 1998 June 11. Note that the CME impulsive acceleration phase coincides well with the flare rise phase.

CME/flare events. In the impulsive acceleration phase, the acceleration rate is $410 \mathrm{~m} \mathrm{~s}^{-2}$ (increments of $1075 \mathrm{~km} \mathrm{~s}^{-1}$ over 44 minutes). In terms of the gravitational acceleration at the surface of the Sun, the $g_{s}$ of $274 \mathrm{~m} \mathrm{~s}^{-2}$, the acceleration rate of this CME is $1.5 g_{s}$ during the impulsive acceleration phase. In the following subsections, we will present the observations of three other CME/flares events using the same rules and illustrations as for the 1998 June 11 event presented above.

\subsection{February 23 Event}

The CME on 1997 February 23 is associated with a GOES B7.2 class flare (background flux at A2 level). The source region of this event is NOAA AR 8019, which was located very close to the east limb (about $\mathrm{N} 25^{\circ}, \mathrm{E} 78^{\circ}$ ) at the time of eruption. Dere et al. (1999) have reported this CME event in detail, concentrating on its helical structure. Here we report on its initiation process and the corresponding flare. Figures $4 a, 4 b$, and $4 c$ display the difference images of $\mathrm{C} 1$ at 01:40 UT (after subtracting the 00:55 UT image, 25302), C1 at 02:10 UT (after subtracting the image at 01:40 UT, $\lambda 5302$ ), and the EIT 2304 image at 02:08 UT, respectively. These three images have been aligned and scaled to the same size.

The CME is initiated at 01:40 UT when its front is seen as a semicircular rim above the active region in the difference image (Fig. $4 a$ ). It has an initial height of $0.47 R_{\odot}$, or $330,000 \mathrm{~km}$ above the limb. This event is also accompanied by an erupting filament as seen in Figure $4 c$ (the eruption process of this filament has been shown in a sequence of EIT $\lambda 304$ images in Dere et al. 1999). The filament trails the CME front, e.g., when the CME front is at $0.50 R_{\odot}$ (Fig. $4 b$ ), the filament is at $0.14 R_{\odot}$ (Fig. $4 c$ ) above the limb. The associated flare, whose position (Fig. 4, plus signs) is determined by the post-flare loops seen in later EIT images, is located at the southern leg of the erupting filament and also close to the southern leg of the CME.

The height-time and velocity-time plots of the CME along with the temporal profiles of the GOES soft X-ray flux are shown in Figure 5. Compared with the event of 1998 June 11, these two events show similar characteristics in terms of CME velocity profile and flare flux profile, although the flare magnitude is much different (B7.2 vs. M1.4). The temporal profile of the X-ray flux can be divided into three phases: a preflare phase of 46 minutes (from $01: 35$ to $02: 21 \mathrm{UT}$ ), a rise phase of 72 minutes (from 02:21 to $03: 33 \mathrm{UT}$ ), and a decay phase of about $9 \mathrm{hr}$ after $03: 33$ UT. The CME velocity profile, which follows the trend of the flare flux, can be divided into an initiation phase, impulsive acceleration phase, and propagation phase, respectively. During the initiation phase (from 01:40 to 02:10 UT), the CME slowly ascended from a height of $0.47-0.50$ $R_{\odot}$; the average speed is only $11 \mathrm{~km} \mathrm{~s}^{-1}$. During the impulsive acceleration phase (from 02:21 to 03:45 UT), the CME speed rapidly increased to a maximum of nearly $900 \mathrm{~km} \mathrm{~s}^{-1}$ at around the time of the flare peak. The acceleration rate during this phase is $210 \mathrm{~m} \mathrm{~s}^{-2}$ or, equivalently, $0.80 g_{s}$. After the time of the flare peak, the CME maintained an almost constant speed of about $800 \mathrm{~km} \mathrm{~s}^{-1}$ until leaving the LASCO field of view.

\subsection{May 16 Event}

The 1997 May 16 CME event is associated with a small GOES B2.1 class flare (background flux at A3 level) and an $\mathrm{H} \alpha$ subflare located at $\mathrm{N} 22^{\circ}$, $\mathrm{W} 69^{\circ}$. Their source region is NOAA AR 8038. In Figure 6, we show the C1 running

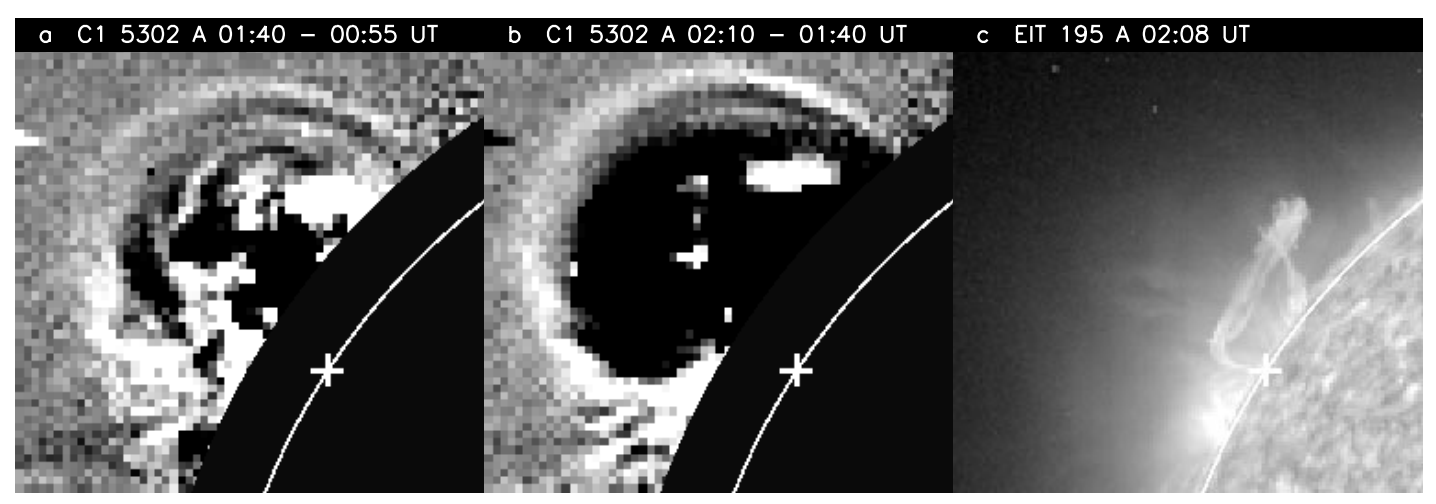

FIG. 4.-Running difference images of $\mathrm{C} 1(a$ and $b)$ and direct EIT $\lambda 304$ image $(c)$ of the CME on 1997 February 23. Their timings are indicated at the top of each panel. The white circle in each panel indicates the $1.0 R_{\odot}$ solar limb. The plus sign indicates the position of the corresponding flare. 

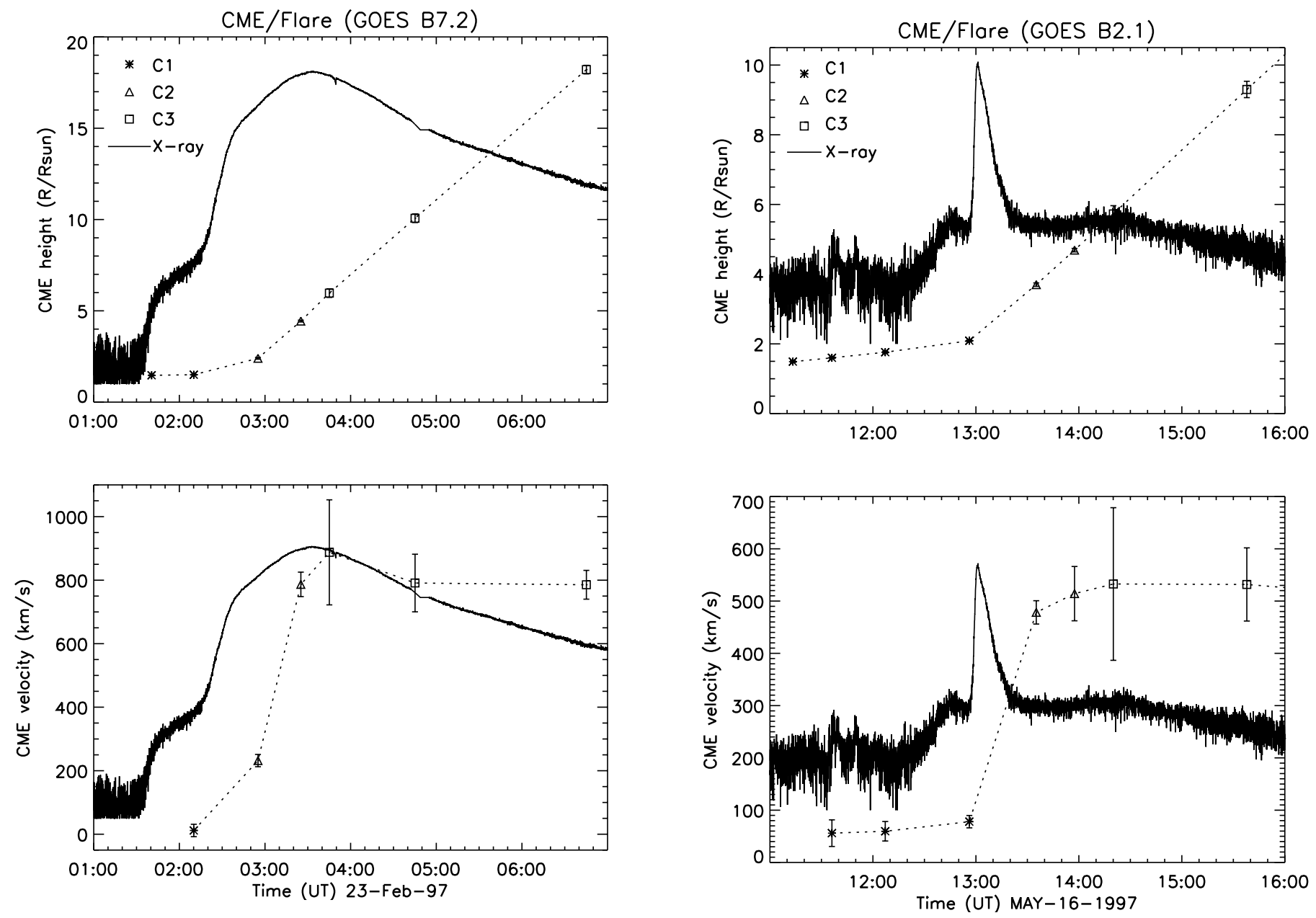

Fig. 5.-Same as Fig. 2, but for the height-time profile of the CME on 1997 February 23 vs. the temporal profile of GOES X-ray flux of the corresponding B7.2 flare.

difference images at 11:13, 11:36, and 12:07 UT. The location of the flare (plus signs) is close to the southern leg of the CME. Using the same conventions as in previous plots, we show the height-time and velocity-time plots of the CME along with the temporal profile of the GOES soft X-ray flux in Figure 7. This flare is a weak and short-duration event
Fig. 7.- Same as Fig. 2, but for the height-time profile of the CME on 1997 May 16 vs. the temporal profile of GOES X-ray flux of corresponding B2.1 flare.

with a total duration of only 15 minutes; it is different from the two previous events, which have hours-long decay phases. Nevertheless, the CME velocity profile can still be decomposed into three phases, as in the two previous events. The CME initiation phase, which occurs before the flare onset, lasts for about $2 \mathrm{hr}$ (from 11:13 to 12:56 UT).

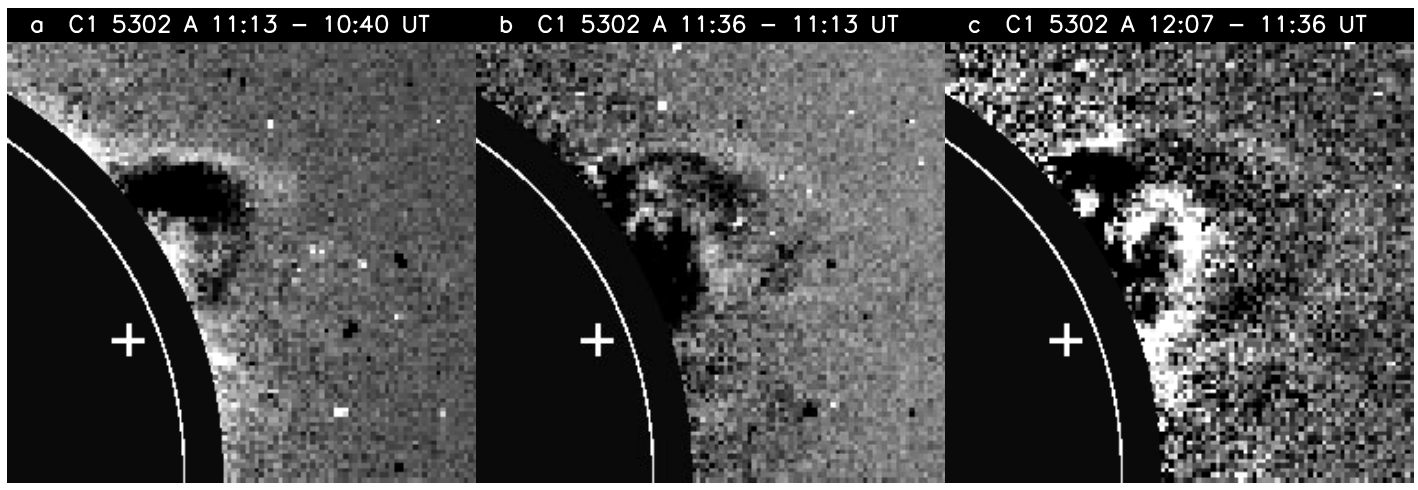

FIG. 6.-Running difference images of $\mathrm{C} 1$ showing evolution of the CME on 1997 May 16. Their timings are indicated at the top of each panel. The white circle in each panel indicates the $1.0 R_{\odot}$ solar limb. The plus sign indicates the position of the corresponding flare. 


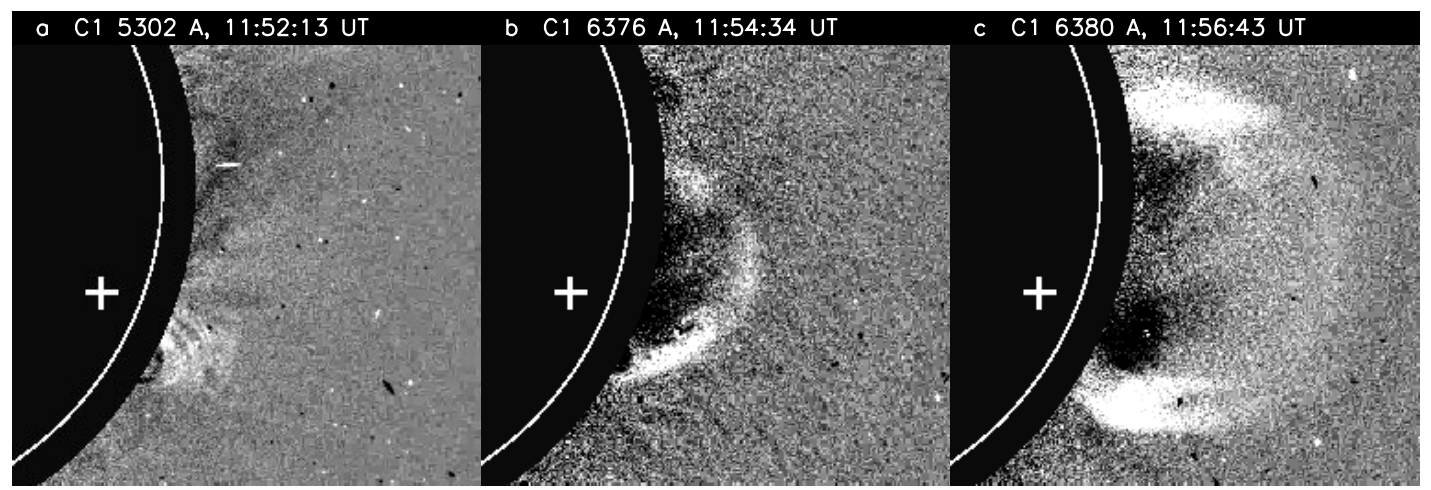

FIG. 8.- Running difference images of $\mathrm{C} 1$ showing evolution of the CME on 1997 November 6. Timings are indicated at the top of each panel. The white circle in each panel indicates the $1.0 R_{\odot}$ solar limb; the plus sign indicates the position of the corresponding flare.

The CME front is first formed at a height of $1.49 R_{\odot}$ and reaches a height of $2.09 \mathrm{UT}$ at the end of the initiation phase; the CME speed during this phase is between 50 and $80 \mathrm{~km} \mathrm{~s}^{-1}$. Note that this CME is not associated with any erupting filament.

Similar to previous events, the CME is impulsively accelerated near the time of the flare. It reached a speed of 478 $\mathrm{km} \mathrm{s}^{-1}$ at 13:35 UT, compared with a speed of $78 \mathrm{~km} \mathrm{~s}^{-1}$ at 12:56 UT. Using these numbers, the acceleration rate is $170 \mathrm{~m} \mathrm{~s}^{-2}$ or, equivalently, $0.61 g_{s}$. This acceleration rate is a lower limit to the real value since the real acceleration time may be much shorter than the LASCO $\mathrm{C} 1$ cadence. After the impulsive acceleration, the CME propagated at a largely constant speed of $500-600 \mathrm{~km} \mathrm{~s}^{-1}$ until its front was out of the field of view of LASCO.

\subsection{November 6 Event}

The CME of 1997 November 6 is associated with a GOES X9.4 flare (background flux at $\mathrm{C} 2$ level) and an $\mathrm{H} \alpha 2 \mathrm{~B}$ flare. Their source region is NOAA AR 8100 , located at $\mathrm{S} 18^{\circ}$, W60 ${ }^{\circ}$. Maia et al. (1999) have presented this event in detail, with a special emphasis on the radio observations. The coronal structure of the active region in EIT images shows a complex configuration with multiple loop systems. Unlike the previous three events, time-lapse EIT and C1 movies of this event do not show a slow ascension phase of these loop systems before the sudden eruption of the CME and flare.

This is an unusually strong event in terms of both CME speed and flare magnitude. In Figure 8, we show consecutively three $\mathrm{C} 1$ difference images all taken within 4 minutes around the impulsive period of the flare. At 11:52:13 UT (Fig. 8a), which is almost at the onset of the impulsive phase of the flare, there is no apparent CME front at all. At 11:54:34 UT (Fig. $8 b$ ) or only 2 minutes later, a semicircular CME front is clearly visible at a height of $1.45 R_{\odot}$. This front rapidly expanded both radially and laterally, reaching a height of $1.88 R_{\odot}$ at 11:56:43 UT. The derived speed along the radial direction is $2150 \mathrm{~km} \mathrm{~s}^{-1}$ between the last two frames. The footpoint of the southern leg is almost fixed in the active region where the flare occurred (the location of the flare [Fig. 8, plus signs] is determined by excessive brightening in EIT images), while the northern leg apparently expanded toward the north to cross the equator.

In Figure 9, the height-time and velocity-time plots of the CME are shown along with the temporal profile of the
GOES soft X-ray flux. In addition, we also show the profile of the hard X-ray flux of HXT in its low channel. It is a very impulsive flare with a rise time of less than 2 minutes and a duration of $\sim 10$ minutes in hard X-rays. Even in soft $\mathrm{X}$-rays, the rise phase of the flare is only a few minutes. The
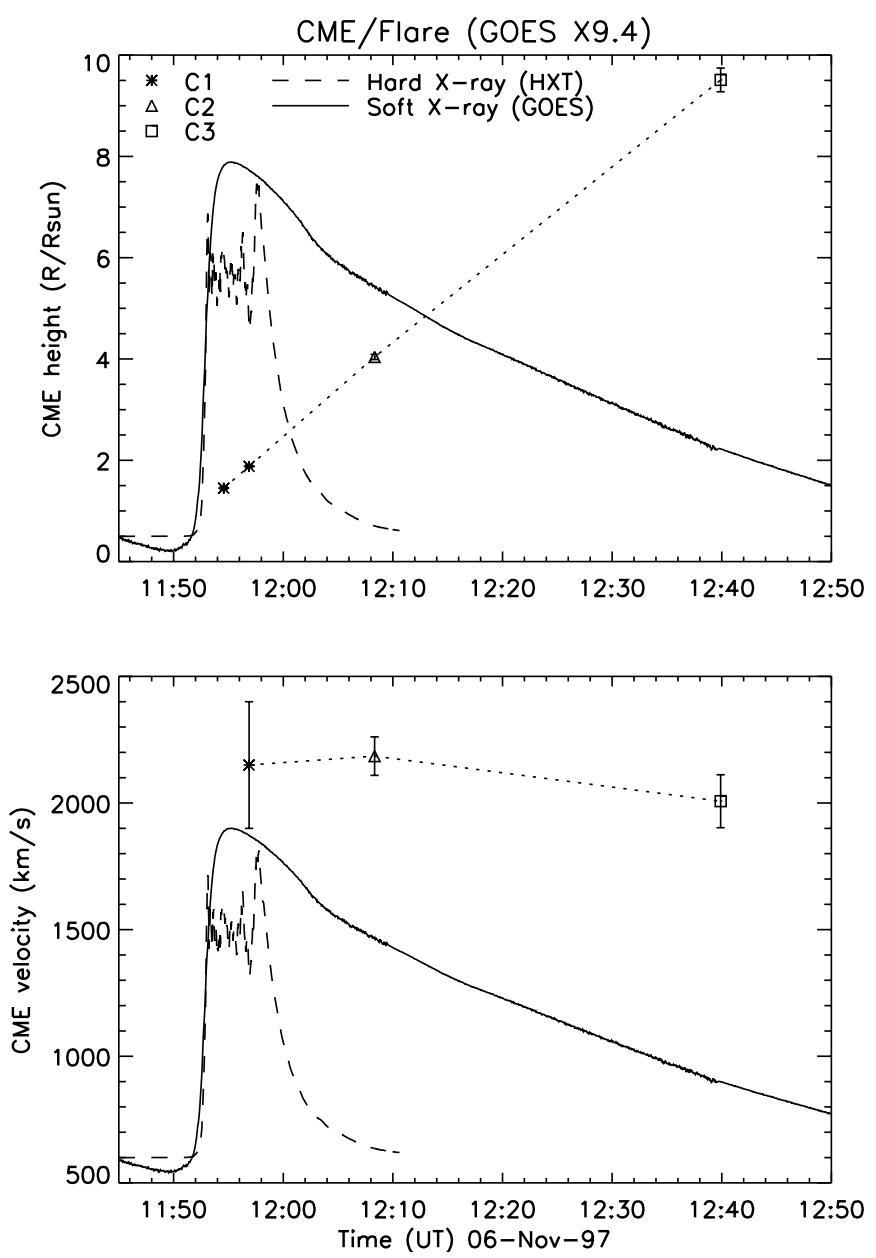

FIG. 9.- Same as Fig. 2, but for the height-time profile of the CME on 1997 November 6 vs. the temporal profile of GOES X-ray flux of the corresponding X9.4 flare. In addition, we show the hard X-ray profile of HXT (dashed line) in both panels. 
decay phase of the GOES flare lasted about 90 minutes. This flare does not show a preflare phase.

Although this CME does not display an initiation phase, the CME is impulsively accelerated during the rise phase of the flare, which is consistent with previous events. The calculated acceleration rate is $7300 \mathrm{~m} \mathrm{~s}^{-2}$ or, equivalently, 26 $g_{s}$, which is significantly larger than that of the previous events. The CME later maintained a constant speed of $\sim 2000 \mathrm{~km} \mathrm{~s}^{-1}$ for $\sim 40$ minutes (until 12:39:54 UT). The CME then gradually slowed down to $1300 \mathrm{~km} \mathrm{~s}^{-1}$ before it went out of the field of view of LASCO at $30 R_{\odot}$.

\subsection{Summary of Observational Results}

One important finding based on previous presentation is that the impulsive acceleration phase of CMEs coincides very well with the rise phase of the accompanying X-ray flares. While the duration of the rise phase of flares can be a few minutes to tens of minutes, the corresponding CMEs always show an apparent speed increment along with the increment of the soft X-ray flux. Before the onset and after the peak of the flare, we do not see significant changes in CME speed. Although we draw this conclusion on the basis of only four events for which we have multiple $\mathrm{C} 1$ observations during the time of the flare, we believe this conclusion is true for other CME/flare events. Indeed, we have investigated a dozen or so other events whose source regions are also close to the limb. Although the $\mathrm{C} 1$ observations of these events do not have sufficient cadence to have multiple observations during the flare rise phase, these events show that after the peak time of the flares, the CMEs have already undergone most of their acceleration, which is consistent with the conclusion.

In Table 1, we summarize the results of the four events studied, the columns representing the date of the event, GOES flare magnitude, flare rise time $t_{r}$, CME acceleration rate $a$ in the impulsive acceleration phase, CME maximum speed $v_{\max }$, CME initiation height $r_{i}$, CME height $r_{f}$ at the end of the acceleration phase, and length or duration $t_{i}$ of the CME initiation phase. The uncertainty of the acceleration rates shall be large not only because of the uncertainty in measuring the exact CME height, but also owing to the uncertainty in the exact timings of onset and termination of the acceleration, which are limited by the observation cadence; we estimate an uncertainty of $30 \%$. The acceleration rates in Table 1 assume a constant acceleration during the impulsive phase. It seems that the stronger the flare, the higher the acceleration rate. But the final speed of a CME should also depend on how long the acceleration will last.

The acceleration of CMEs appears to stop at about the peak time of the soft X-ray flare. For each event there is a final height above which no significant acceleration can be observed. The column of $r_{f}$ in Table 1 represents such a final height. It ranges from 1.8 to $4.6 R_{\odot}$ for these events. It has been well known that fast CMEs display a largely constant speed in white-light coronagraphs (MacQueen \& Fisher 1983; Sheeley et al. 1999; Andrews \& Howard 2001; St. Cyr et al. 2000). This may be understandable in view of our results that these CMEs are impulsively accelerated in the low corona during the rise phase of flares. Another important parameter is the initiation height of the CME. In Table 1, the column of $r_{i}$ indicates the height at which the CME front is first formed. It ranges from 1.3 to $1.5 R_{\odot}$ for these events. Apparently, these CMEs do not form very close to the surface.

\section{DISCUSSION}

\subsection{Initiation Phase of CMES}

The "initiation phase" used in the presentation above refers to a period of slow ascension of CMEs ahead of the impulsive acceleration phase. Three of the four CMEs studied display the initiation phase as defined. The period of this phase (see column $t_{i}$ of Table 1) may last from half an hour to almost $2 \mathrm{hr}$ from event to event. In this phase, the CME front is first formed and then undergoes a slow expansion with a speed from 5 to $80 \mathrm{~km} \mathrm{~s}^{-1}$. These characteristics are consistent with low coronal observations of the 1980 August 5 event (Low, Munro, \& Fisher 1982), one of the first uses of data from the ground-based K-coronameter; unfortunately, the subsequent evolution of this event could not be observed. The initiation phase can be easily differentiated from the impulsive acceleration phase on the basis of the slope or acceleration rate in the velocity-time plot of a CME; the acceleration rate can be 2 orders of magnitude different between the two phases. Furthermore, the observations show that the onset of the accompanying flare largely marks the end of the initiation phase but marks the onset of the impulsive acceleration phase. Therefore, we argue that the initiation phase represents a distinct evolution stage of a CME. On theoretical grounds, it has been suggested that a large-scale coronal structure may undergo a stage of quasi-static evolution before reaching a critical point at which violent magnetic activity may onset (Low et al. 1982; Hundhausen 1993). It is possible that the initiation phase leads to the eruption of CMEs as well as flares. However, we should note that one of the events studied (1997 November 6) does not display an initiation phase. This event corresponds to an intensive X-ray flare (X9.4) and extremely large acceleration rate $\left(7300 \mathrm{~m} \mathrm{~s}^{-2}\right)$. It may have a different evolution behavior from those events displaying an initiation phase.

The linear extrapolation method has been used to determine the CME's onset time in early papers. This extrapolation method is not accurate since the assumptions behind this method are not true. Neither is the CME in constant

TABLE 1

PropertiES OF CMES AND FLARES

\begin{tabular}{cccccrrr}
\hline \hline Event & GOES & $\begin{array}{c}t_{r} \\
(\text { minutes })\end{array}$ & $\begin{array}{c}\mathrm{a} \\
\left(\mathrm{m} \mathrm{s}^{-2}\right)\end{array}$ & $\begin{array}{c}v_{\max } \\
\left(\mathrm{km} \mathrm{s}^{-1}\right)\end{array}$ & $\begin{array}{c}r_{i} \\
\left(R_{\odot}\right)\end{array}$ & \multicolumn{1}{c}{$\begin{array}{c}r_{f} \\
\left(R_{\odot}\right)\end{array}$} & $\begin{array}{r}t_{i} \\
\text { (minutes) }\end{array}$ \\
\hline 1998 Jun 11...... & M1.4 & 30 & 410 & 1143 & 1.34 & 4.64 & 23 \\
1997 Feb 23 ...... & B7.2 & 72 & 210 & 887 & 1.47 & 4.44 & 30 \\
1997 May 16..... & B2.1 & 5 & $>170$ & 532 & 1.49 & 3.70 & 103 \\
1997 Nov 6 ....... & X9.4 & 6 & 7300 & 2185 & $<1.45$ & $\sim 1.88$ & 0 \\
\hline
\end{tabular}


speed in the low corona, nor does the CME launch from solar surface. Using this extrapolation method, it has been suggested that the onset time of a CME coincides with a flare precursor, which is a small flare tens of minutes earlier than the main flare (Harrison et al. 1985, 1990; Simnett \& Harrison 1985; Harrison 1986). What we find is that the CME is initiated earlier than the onset of the main flare but that it possibly corresponds to a preflare phase, as seen as a small bump of X-ray flux ahead of the rise phase of the flare. We are not sure whether the preflare phase seen in our events is exactly the so-called precursor flare in those studies. We need to emphasize that the significant acceleration of the CME starts almost simultaneously with the flare onset, not with any flare precursor feature. While the constant speed fitting to the early evolution of CMEs is not appropriate, we further point out that even a constant acceleration rate fitting is not adequate for some events, since CME's initiation phase is in sharp difference in acceleration rate from its impulsive acceleration phase. St. Cyr et al. (1999) have carried out an extensive study on the initial evolution of 55 CMEs using combined MK3 and SMM observations and found a wide range of acceleration rates (from -218 to $3270 \mathrm{~m} \mathrm{~s}^{-2}$ ). They did not differentiate the initiation phase from the impulsive acceleration phase of CMEs, largely owing to the limitation of cadence.

In terms of the outstanding argument about relative onset time between CMEs and flares, our observations suggest an intriguing scenario: the onset time of CME's initiation phase starts earlier than the onset time of the flare by tens of minutes, while the onset time of CME's impulsive acceleration phase starts almost simultaneously with the flare's onset time. These facts simply reject the view that the flare is the cause of the CME. On the other hand, however, we cannot simply conclude that the CME is the cause of the flare. We discuss this issue further in the following section.

\subsection{Impulsive Acceleration Phase of CMEs}

We find that the impulsive acceleration phase of a CME coincides very well with the rise phase of the accompanying soft X-ray flare. It appears that the onset of the CME's impulsive acceleration phase occurs almost simultaneously with the flare's onset time, and the acceleration stops at a time close to the peak time of the X-ray flux. The CME then moves outward in the middle and/or high coronae at more or less a constant speed, while the flux of the flare decreases gradually. The above description generally fits the observations of the four events, although we point out that the exact timing of onset and termination of the impulsive acceleration phase is subject to an uncertainty of a few minutes, the limitation of observation cadence. Some early studies have already demonstrated that CMEs start almost simultaneously with flares (Maxwell, Dryer, \& McIntosh 1985; Dryer 1994). Recently, Hundhausen (1999) also showed the close temporal relation between CMEs and flares, but he suggested that flare onset lags behind the $\mathrm{CME}$ onset by at least a few minutes. Our observations are consistent with these findings if we do not draw clear separation between the initiation phase and the impulsive acceleration phase.

We now discuss the implications of these observations. In the past, each of the following three scenarios has been proposed: the flare causes the CME, the CME causes the flare, or the CME and the flare are different manifestations of the same magnetic process in the corona. The flare-cause-
CME scenario argues that a sudden increment of thermal pressure in the low corona, as evidenced by the observed increment of density and temperature in the flare, produces fast blast waves and certain CMEs (Parker 1961; Wu, Dryer, \& Han 1983; Maxwell et al. 1985). This traditional scenario has been largely challenged by detailed observations, e.g., the disparity in size scales and asymmetry in locations between flares and CMEs (Harrison 1986, 1995; Kahler et al. 1989; Harrison et al. 1990). As mentioned above, the finding that the initiation phase starts tens of minutes earlier than the flare onset also strongly rejects this scenario. Furthermore, we find that the height range over which CME acceleration takes place, e.g., from 1.3 to 4.6 $R_{\odot}$, is much higher than the observed height of the flare region. Considering that any transient front such as a blast wave or shock wave created by a thermal pressure impulse should decrease in speed when it detaches the region of enhanced pressure, the acceleration-height range of CMEs also argues against the thermal pressure-driven scenario. In contrast to the problematic flare-cause-CME scenario, the CME-cause-flare scenario suggests that the flare may be the consequence or by-product of the CME (Kahler 1992; Hundhausen 1999). It seems a reasonable choice if there is a true cause-effect relationship between CMEs and flares.

However, there is an another alternative to interpret the close relationship between CMEs and flares. There is no strict cause-effect relationship between them; they are merely different manifestations or consequences of the same coronal process (Harrison 1995). Many of the observational facts can be used to support both this common-cause scenario or CME-cause-flare scenario, which makes the justification of a correct scenario difficult. It is even more difficult to differentiate the two scenarios if one views the magnetic process that leads to the CMEs and flares as a part of the CME itself. Nevertheless, we think that the common-cause scenario is a better interpretation of the observations for the following reasons: first, the close temporal correlation between the CME's impulsive acceleration phase and the flare's rise phase does not show a clear cause-effect relationship between them; second, there is no correlation between energetics of CMEs (in terms of speed) and flares (in terms of peak flux) (e.g., Harrison 1995). Fast CMEs can be associated with weak flares, while intensive flares can be associated with no CMEs. Since any cause-effect relationship would imply a correlation in energetics between CMEs and flares, the common-cause scenario does not cause possible confusion. In short, we believe that CMEs and associated flares have a strongly coupled relationship but do not cause one another.

In order to explain those CMEs that display an impulsive acceleration phase as well as an initiation phase, theoretical models need to incorporate a two-step evolution of magnetic structure in the corona. The first step is a state of quasi-static expansion, while the second step is in a much more violent state, which induces magnetic force to drive CMEs and accelerate particles or heat plasma to cause flares. Various theoretical models have been proposed to explain CMEs and flares that involve magnetic reconnection. The model of Anzer \& Pneuman (1982), which suggests that the reconnection forming the post-flare loops may drive CMEs, cannot be correct since CME acceleration occurs during the flare rise phase while the formation of post-flare loops corresponds to the decay phase of flares. The flux-rope models, which relate the CME to two-ribbon 
flares or post-flare loops (Forbes \& Priest 1995; Amari et al. 2000; Lin \& Forbes 2000), usually require spatial symmetry between CMEs and flares. However, that symmetry is not supported by observations. The recently proposed "breakout" model (Antiochos 1998; Antiochos, DeVore, \& Klimchuk 1999), which exploits a multipolar magnetic topology, seems to be more consistent in this matter. In the "breakout model," where multiple flux systems exist, the flare may not be strictly required to occur right beneath the center of the CME. Also note that some models could explain the acceleration of CMEs without necessarily involving magnetic reconnection (Low \& Hundhausen 1995; Low 1996; Chen 1996; Wu 1997).

\subsection{Other Considerations}

The four CMEs presented in this paper are flareassociated events that display an impulsive acceleration. It is important to find out whether the impulsive acceleration is always the characteristic of flare-associated CMEs, although the flare could be a minor one. Using groundbased K-coronameter observations of 12 CMEs, MacQueen \& Fisher (1983) found that flare-associated CMEs exhibit high speed and thus impulsive acceleration, while the filament-associated events show low speed and gradual acceleration. Using LASCO $\mathrm{C} 2 / \mathrm{C} 3$ observations but without checking into association with surface phenomena, Sheeley et al. (1999) studied the detailed kinematic behavior of CMEs and classified them into two principle types: impulsive and gradual CMEs. Andrews \& Howard (2001) made similar classifications of CMEs. We have examined more than a dozen other CMEs (not presented here) also originated close to the limb; with EIT, C1, C2, and C3 observations, it seems true that CMEs displaying impulsive acceleration are always closely associated with flares. Hundhausen (1999) showed a fast CME event (1988 March 18) without a flare associated; however, the GOES flux background during the observation was at a $\mathrm{C} 2$ level, which may overshadow a minor flare. On the other hand, we notice that CMEs displaying no impulsive acceleration are not associated with flares. One example of this kind of CME is the event on 1997 October 19 (see details in Dere et al. 1999), which gradually increases its speed. During a period of $29 \mathrm{hr}$ seen in the LASCO, this CME reached a speed of only $450 \mathrm{~km} \mathrm{~s}^{-1}$. The acceleration rate turns out to be only $5 \mathrm{~m} \mathrm{~s}^{-2}$ (equivalently $0.018 g_{s}$ ), which is significantly smaller than in the impulsive events presented in this paper. This event is not associated with any flare (background GOES flux is at a B1 level); it is associated with a filament eruption.

On the basis of the above discussions, it seems that the occurence of flares is an important indicator of how a CME has evolved. Destabilization of the large-scale magnetic structure in the corona initiates the process. The magnetic structure continuously undergoes a quasi-static evolution during which the CME displays a slow ascension, or socalled initiation phase. Without reaching a critical point, the CMEs would continue the slow ascension and eventually merge with the background solar-wind flow. In this way, it forms the gradual CME. In many cases, however, the critical point is reached, and the resulted violent magnetic activity produces both fast CMEs and flares. In additional to these two possibilities, there may exist another class of CME/flare events that do not show an initiation phase, e.g., the 1997 November 6 event presented in this paper.

\section{CONCLUSIONS}

In this paper, we have investigated the detailed temporal relationship between CMEs and associated flares for four well-observed events. The information on the early initiation and acceleration of CMEs, which was not available in previous space-based observations, has been obtained using EIT and LASCO observations. The source regions of these events are close enough to the limb for the initial CME fronts to be seen in EIT and LASCO/C1. Furthermore, these events happen to have multiple $\mathrm{C} 1$ observations during their acceleration process. The advantage of this selection is to straightforwardly determine the onset time of CMEs and track the initiation and acceleration of CMEs without resorting to any extrapolation. Extrapolation methods, which are based on some inappropriate assumptions, such as CMEs have a constant speed behind the occulter and CMEs are initiated at a zero altitude, have caused controversial results.

On the basis of their velocity-time profiles, the kinematic evolution of three of the four CMEs studied can be described in a three-phase scenario: the initiation phase, impulsive acceleration phase, and propagation phase. The initiation phase is characterized by a slow ascension with a speed less than $80 \mathrm{~km} \mathrm{~s}^{-1}$ for a period of tens of minutes. The initiation phase of a CME always occurs before the onset of the associated flare but sometimes corresponds with a small bump of soft X-ray flux in a preflare phase. Following the initiation phase, the CME displays an impulsive acceleration phase, which starts almost simultaneously with the flare onset time and coincides very well with the flare's rise phase lasting for a few to tens of minutes. The acceleration of CME ceases near the peak time of the soft $\mathrm{X}$-ray flare. The CME then undergoes a propagation phase, which is characterized by a constant speed or slowly decreasing in speed. The acceleration rate in the impulsive acceleration phase of these three CMEs is in the range of $100-500 \mathrm{~m} \mathrm{~s}^{-2}$. The CMEs are initiated at a height of 1.3-1.5 $R_{\odot}$ and accelerated until the height of 3.7-4.7 $R_{\odot}$. One CME (on 1997 November 6, associated with an X9.4 flare) does not show an initiation phase. It has an extremely large acceleration rate of $7300 \mathrm{~m} \mathrm{~s}^{-2}$. It also has a lower initiation height $\left(<1.45 R_{\odot}\right)$ and a lower maximum acceleration height $\left(\sim 1.88 R_{\odot}\right)$.

Our results reject the scenario that CMEs are driven by flare-induced thermal pressure, since CMEs (three of the four events studied) are initiated before the onset of the associated flares. The initiation phase of CMEs may be caused by the destabilization and quasi-static evolution of the large-scale magnetic structure in the corona. If a critical point is reached during the quasi-static evolution, violent magnetic activity may be triggered that induces the magnetic force to drive CMEs, while simultaneously accelerate particles or heat plasma to cause flares. In this scenario, CMEs and flares are two different manifestations of the same magnetic process in the corona; they have a strongly coupled relationship but not a cause-effect one.

This work is funded by NASA. J. Zhang thanks J. Klimchuk, A. Vourlidas, P. Subramanian, and Paul Reiser for valuable discussions. SOHO is a project of international cooperation between ESA and NASA. YOHKOH is a project of international cooperation between Japan, US, and UK. 


\section{REFERENCES}

Amari, T., Luciani, J. F., Mikic, Z., \& Linker, J. 2000, ApJ, 529, L49

Andrews, M. D., \& Howard, R. S. 2001, Space Sci. Rev., 95, 147

Antiochos, S. K. 1998, ApJ, 502, L181

Antiochos, S. K., DeVore, C. R., \& Klimchuk, J. A. 1999, ApJ, 510, 485

Anzer, U., \& Pneuman, G. W. 1982, Sol. Phys., 79, 129

Brueckner, G. E., et al. 1995, Sol. Phys., 162, 357

Chen, J. 1996, J. Geophys. Res., 101, 27499

Delaboudinière, J.-P., et al. 1995, Sol. Phys., 162, 291

Dere, K. P., Brueckner, N. A., Howard, R. A., Michels, D. J., \& Delaboudinière, J. P. 1999, ApJ, 516, 465

Dryer, M. 1994, Space Sci. Rev., 67, 363 1996, Sol. Phys., 169, 421

Fisher, R. R., \& Poland, A. I. 1981, ApJ, 246, 1004

Forbes, T. G., \& Priest, E. R. 1995, ApJ, 446, 377

Gosling, J. T. 1993, J. Geophys. Res., 98, 11, 18937

Harrison, R. A. 1986, A\&A, 162, 283 1995, A\&A, 304, 585

Harrison, R. A., Hildner, E., Hundhausen, A. J., Sime, D. G., \& Simnett, G. M. 1990, J. Geophys. Res., 95, 917

Harrison, R. A., Waggett, P. W., Bentley, R. D., Phillips, K. J., \& Bruner, M. 1985, Sol. Phys., 97, 387

Hundhausen, A. J. 1993, J. Geophys. Res., 98, 13177

1999, in The Many Faces of the Sun, ed. K. Strong, et al. (New York: Springer), 143

Kahler, S. M. 1992, ARA\&A, 30, 113

Kahler, S. W., Sheeley, N. R., Jr., \& Liggett, M. 1989, ApJ, 344, 1026
Lin, J., \& Forbes, T. G. 2000, J. Geophys. Res., 105, 2375

Low, B. C. 1996, Sol. Phys., 167, 217

Low, B. C., \& Hundhausen, J. R. 1995, ApJ, 443, 818

Low, B. C., Munro, R. H., \& Fisher, R. R. 1982, ApJ, 254, 335

MacQueen, R. M., \& Fisher, R. R. 1983, Sol. Phys., 89, 89

Maia, D., Vourlidas, A., Pick, M., Howard, R., Schwenn, R., \& Magalhaes, A. 1999, J. Geophys. Res., 104, 12507

Maxwell, A., Dryer, M., \& McIntosh, P. 1985, Sol. Phys., 97, 401

Munro, R. H., Gosling, J. T., Hildner, E., MacQueen, R. M., Poland, A. I., \& Ross, C. L. 1979, Sol. Phys., 61, 201

Parker, E. N. 1961, ApJ, 133, 1014

Plunkett, S. P., et al. 1997, Sol. Phys., 175, 699

Schwenn, R., et al. 1997, Sol. Phys., 175, 667

Sheeley, N. R., Jr., Howard, R. A., Koomen, M. J., \& Michels, D. J. 1983, ApJ, 272, 349

Sheeley, N. R., Jr., Walters, H., Wang, Y.-M., \& Howard, R. A. 1999, J. Geophys. Res., 104, 24739

Simnett, G. M., \& Harrison, R. A. 1985, Sol. Phys., 99, 291

St. Cyr, O. C., Burkepile, J. T., Hundhausen, A. J., \& Lecinski, A. R. 1999, J. Geophys. Res., 104, 12493

St. Cyr, O. C., et al. 2000, J. Geophys. Res., 105, 18169

St. Cyr, O. C., \& Webb, D. F. 1991, Sol. Phys., 136, 379

Webb, D. F., \& Hundhausen, A. J. 1987, Sol. Phys., 108, 383

Wu, S. T. 1997, Sol. Phys., 170, 265

Wu, S. T., Dryer, M., \& Han, S. M. 1983, Sol. Phys., 84, 395 